\title{
How well do ordinary Americans forecast the growth of COVID-19?
}

Madison Fansher ${ }^{1 *}$, Tyler J. Adkins ${ }^{1 *}$, Richard L. Lewis ${ }^{1,2,3}$, Aysecan Boduroglu ${ }^{4}$, Poortata Lalwani $^{1}$, Madelyn Quirk ${ }^{1}$, Priti Shah ${ }^{1}$, John Jonides ${ }^{1}$.

${ }^{1}$ Department of Psychology, University of Michigan, Ann Arbor, MI, 48109

${ }^{2}$ Department of Linguistics, University of Michigan, Ann Arbor, MI, 48109

${ }^{3}$ Weinberg Institute for Cognitive Science, University of Michigan, Ann Arbor, MI, 48109.

${ }^{4}$ Department of Psychology, Boğaziçi University, Istanbul, Turkey

*These authors contributed equally to this work. 


\begin{abstract}
Across four experiments, we found that Americans misestimated the growth of COVID-19 cases in the U.S. and that these estimates were in turn related to people's forecasts of the duration of social distancing orders, and their own future adherence to these orders. We also investigated 5 how mode of data presentation influences forecasting of future cases by showing participants data on the number of COVID-19 cases from a five-week period in either graphical or tabular form. We found that people shown tables produced more accurate forecasts compared to people shown line-graphs of the same data; yet, people shown line-graphs were more confident in their estimates. These findings suggest that graphs engender false-confidence in the accuracy of 10 forecasts, that people's forecasts of future cases may have important implications for their attitudes concerning social distancing, and that tables are better than graphs for informing the public about the trajectory of COVID-19.
\end{abstract}


How well do ordinary Americans forecast the growth of COVID-19?

On March 18, 2020, CNN broadcast an aerial view of a beach on the west coast of Florida where large numbers of young people were elbow-to-elbow celebrating their spring breaks from class. Even though the potential danger of the coronavirus was well-publicized then, these young people and the nation at large seemed not to be taking the threat of the virus seriously, as people still frequented restaurants, bars, and other social gatherings. How is it that otherwise intelligent and informed individuals could engage in such close social interaction at a time when the proliferation of cases of COVID-19 both in other countries and in the United States was making daily, indeed hourly, headlines?

There are many variables that influence behavior of this sort, including feelings of invincibility, sense of distance from the virus, age of the individuals, and others. We argue that one influential variable is a fundamental misunderstanding of the rate of growth of an exponential function (at least early on in the growth of the disease) as it relates to disease incidence, deaths due to the disease, and the impact of the disease on the health care

15 system. Prior research has shown that people consistently underestimate exponential growth ${ }^{1-3}$ and are overconfident in their understanding of exponential functions, also known as exponential growth bias ${ }^{4}$.

Consider this one problem: As of March ${ }^{\text {st }}$, the CDC reported that there had been 75 cases of COVID-19 and 1 death in the United States. On March $18^{\text {th }}$, there were 7,038 confirmed cases and 97 deaths due to the disease. How many cases and deaths would there be on March $25^{\text {th }}$ ? April $1^{\text {st? }}$ April $8^{\text {th }}$ ? To the extent that this kind of forecasting is difficult and inaccurate, it may lead to lack of caution among many. 
To test whether there is a gross misestimation of exponential growth of the disease and whether different modes of presenting information about growth might influence judgments, we administered an online survey on March $27^{\text {th }}, 2020$ to 817 people in the United States (U.S.). We presented participants with cumulative growth trends for COVID-19 cases in the U.S. in the preceding five weeks from February $29^{\text {th }}$ to March $27^{\text {th }}$ (Fig. 1A). Half of participants viewed these data in tables (Table group) and half were shown these data as line graphs (Graph group). Only five data points were shown to participants as prior work has shown that the more data that are available to participants, the worse they are at forecasting exponential growth ${ }^{2,3}$. After viewing the data, participants predicted the total number of future cases in the U.S. 3 (March $\left.1030^{\text {th }}\right), 6\left(\right.$ April $\left.2^{\text {nd }}\right)$, and $9\left(\right.$ April $\left.5^{\text {th }}\right)$ days later.

Due to the dynamic nature of the current COVID-19 outbreak, it has been argued that forecasting the future of the disease with accuracy is uniquely difficult ${ }^{5}$ and research from the first week of the pandemic showed that individuals significantly underestimated their personal risk compared to that of the average American, average person in their state, and their

15 neighborhood ${ }^{6}$. We therefore predicted that our participants would underestimate the future trajectory of COVID-19 cases in the U.S. Additionally, previous studies have found that presenting data in a graphical rather than tabular form significantly reduces forecasting errors ${ }^{5}$. We therefore predicted that the Graph group would produce the most accurate forecasts of COVID-19 cases at future dates. All analyses were conducted using Bayesian Hierarchical 20 Linear Models to account for nesting of responses within States. 
A

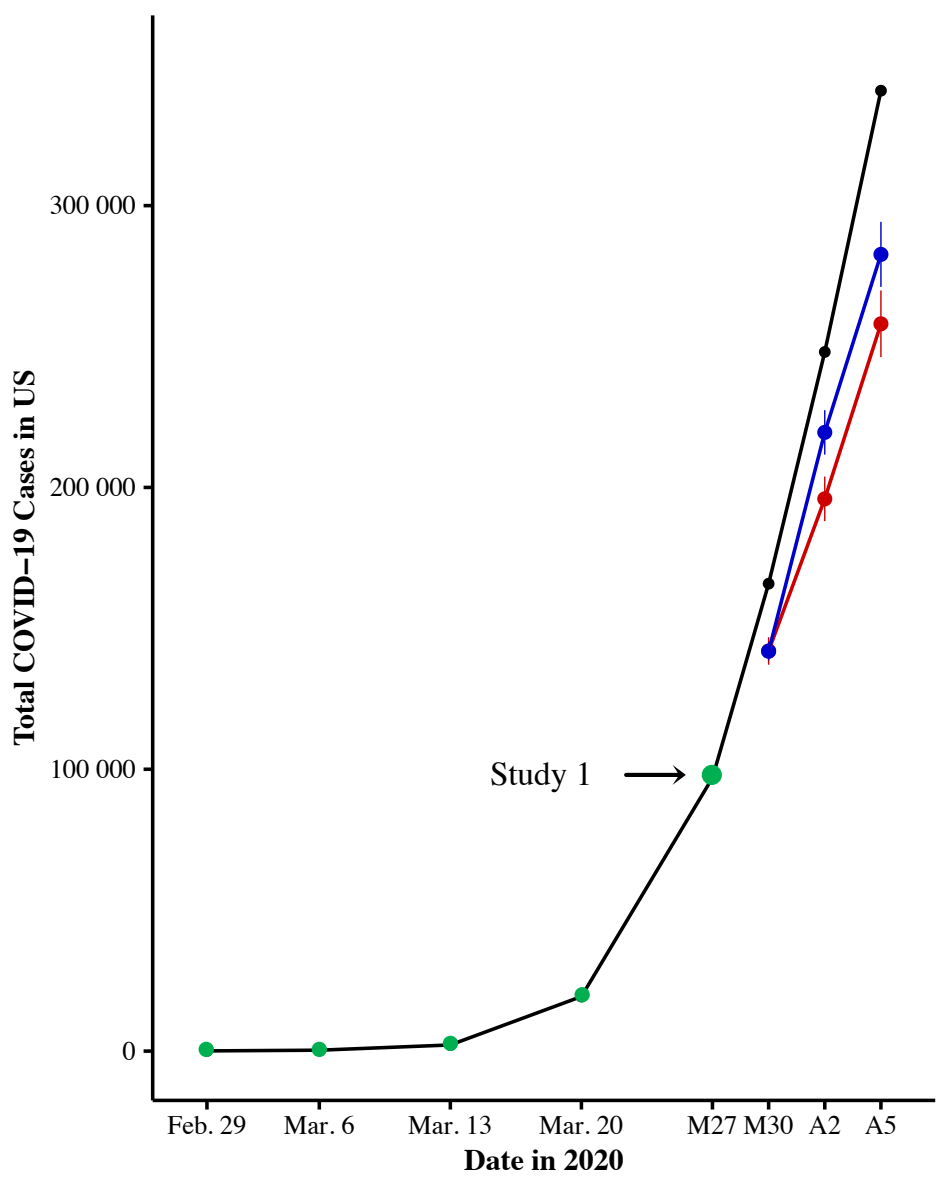

B

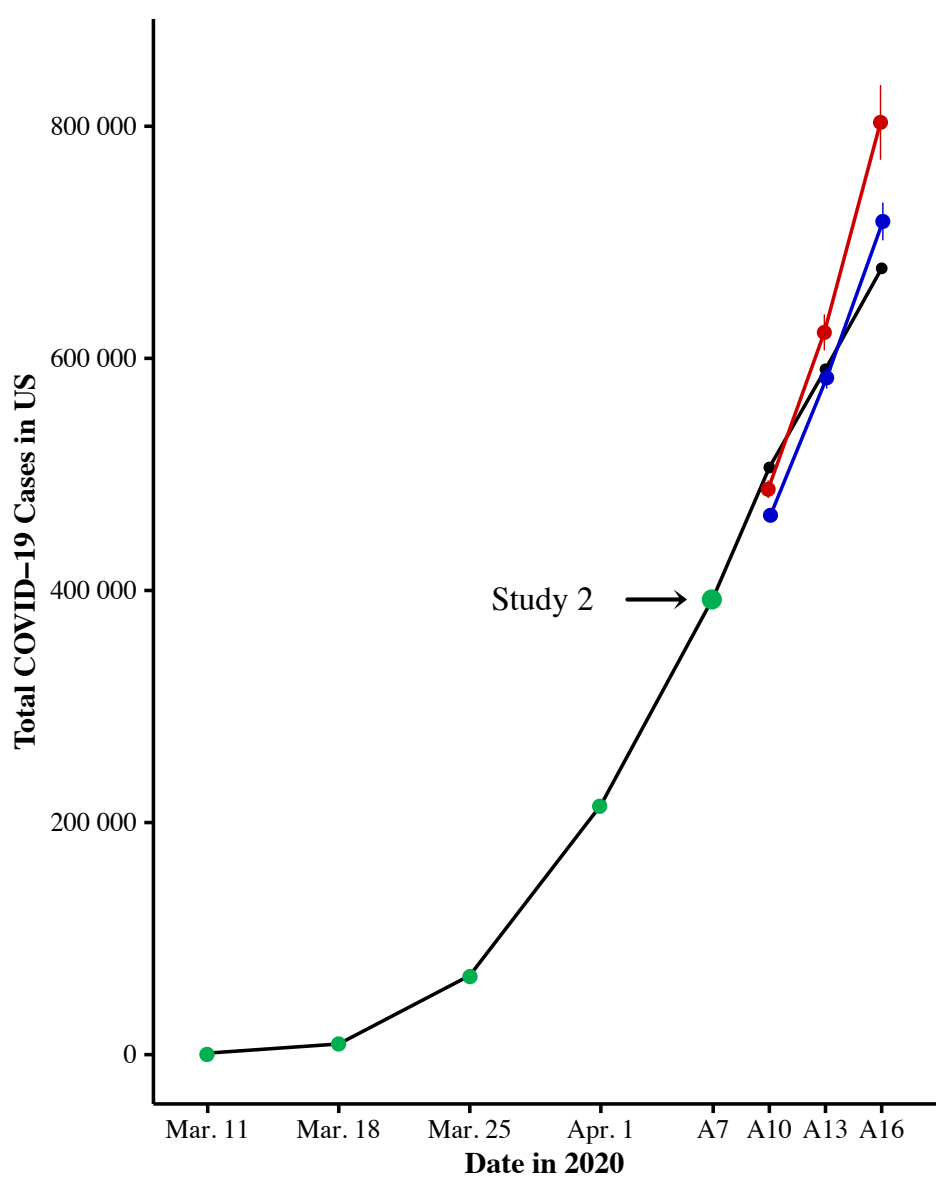

Table - Graph

Figure 1. Results from Study 1 and Study 2. People who viewed graphs of COVID-19 growth in the US produced less accurate forecasts compared to people who viewed the same data in tables. Study 1 was conducted on March $27^{\text {th }}, 2020$, and Study 2 was conducted on April $7^{\text {th }}, 2020$. The black lines with green dots signify the data that participants viewed before making their forecasts. Note that the number cases appear to be growing exponentially in the data presented in Study 1 whereas the growth appears to become more linear in the data presented in Study 2. The black lines after the study dates reflect the total number of confirmed COVID-19 cases in the US according to worldometers.info, accessed on April $27^{\text {th }}, 2020$. The colored lines show the mean forecasts for participants in the graph groups (red) and table groups (blue).

On average, participants underestimated the number of COVID-19 cases in the U.S. on March $30^{\text {th }}\left[\mu_{o b s} \approx 142 \mathrm{k}\right.$, Truth $\left.\approx 164 \mathrm{k}\right]$, April $2^{\text {nd }}\left[\mu_{o b s} \approx 209 \mathrm{k}\right.$, Truth $\left.\approx 245 \mathrm{k}\right]$, and April $5^{\text {th }}\left[\mu_{o b s}=271 \mathrm{k}\right.$, Truth $\left.=339 \mathrm{k}\right]($ Fig. 1A). Critically, we found that the Graph group forecasted fewer future cases than the Table group $\left[\beta_{\mathrm{T}-\mathrm{G}}=21.7 \mathrm{k}, \mathrm{HDI}_{89 \%}=[5.68 \mathrm{k}, 37.7 \mathrm{k}], p d=98.5 \%\right]$ 
(Fig. 1A). Further, we found that the Table group forecasted greater (hence more accurate) increases in cases from March $30^{\text {th }}$ to April $2^{\text {nd }}$ than the Graph group [Fig. $1 \mathrm{~A} ; \beta_{\mathrm{A} 2-\mathrm{M} 30 * \mathrm{~T}-\mathrm{G}}=$ $\left.44.4 \mathrm{k}, \mathrm{HDI}_{89 \%}=[7.28 \mathrm{k}, 81.0 \mathrm{k}], p d=97.3 \%\right]$. Although tables produced the most accurate estimates, people shown graphs were more confident in their inaccurate predictions than people shown tables [Fig. 2A; $\left.\mathrm{CI}_{95 \%}=[-9.46,-5.24], p<.001\right]$. The findings from this study indicate that Americans generally underestimated the growth of COVID-19 but were more accurate when they were presented with epidemiological data in tables rather than graphs. It is important to note that these results were found at a time when the growth of COVID-19 cases in the U.S. had begun to accelerate rapidly, the news was completely dominated by the pandemic, and exponential growth curves were common fixtures in news reports.
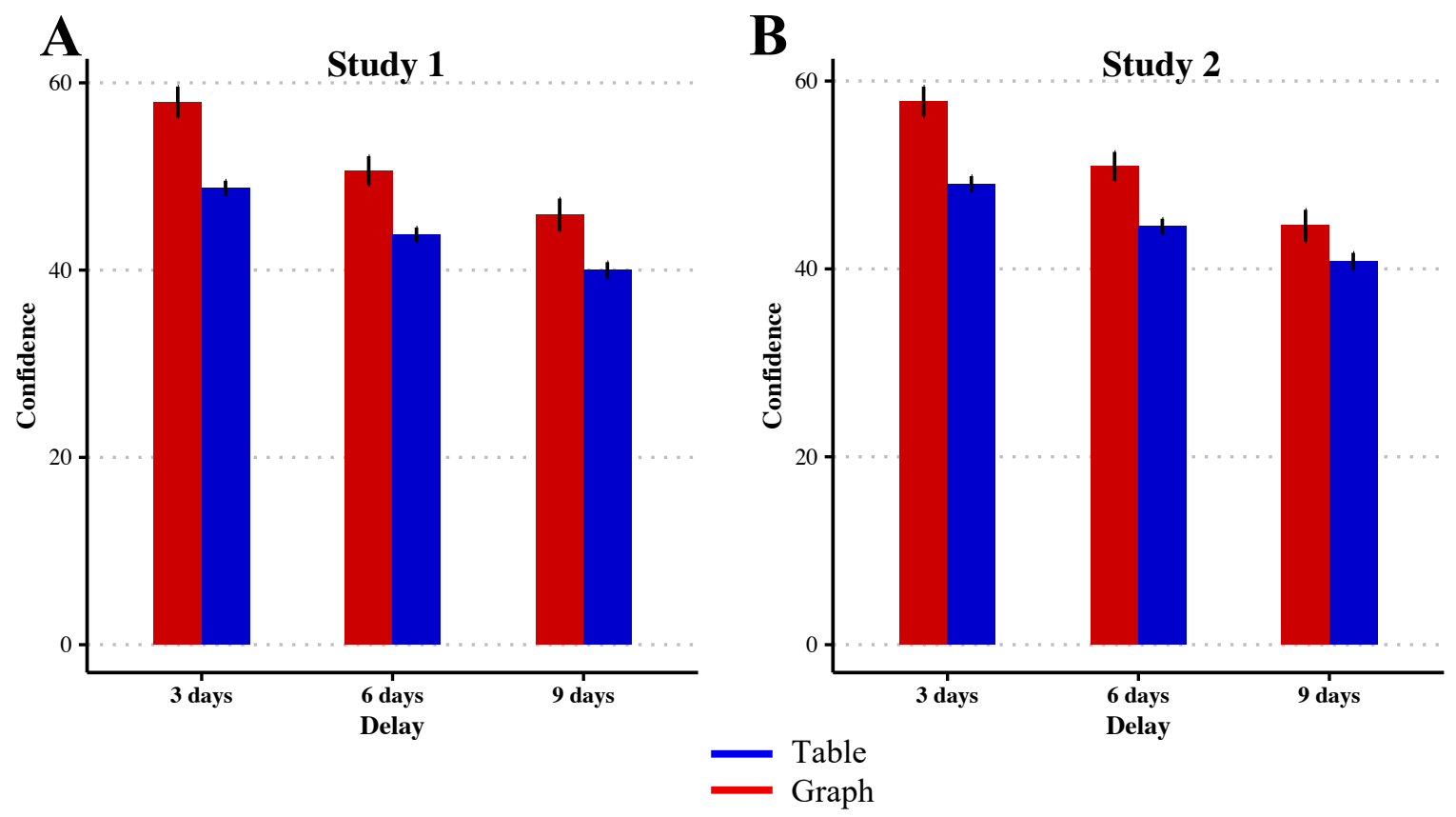

Figure 2. Self-reported confidence for predictions made 3, 6, and 9 days into the future. Here we plot mean confidence and standard errors of the mean confidence for each estimation and illustrate confidence by group. We observe that people shown graphs were more confident in their estimates in Studies 1 and 2, and that confidence decreased over time such that participants were less confident about their 6-day estimate than their 3-day estimate $\left[\mathrm{S} 1: \mathrm{CI}_{95 \%}=[-7.85,-3.02], p<.001 ; \mathrm{S} 2: \mathrm{CI}_{95 \%}=[-7.42,-2.47], p<.001\right]$ and less confident about their 9day estimate than their 6-day estimate $\left[\mathrm{S}_{1}: \mathrm{CI}_{95 \%}=[-6.63,-1.69], p<.001 ; \mathrm{S} 2: \mathrm{CI}_{95 \%}=[-6.38 .-1.54], p<\right.$ 
.001]. This observed decrease in confidence with increased forecasting interval illustrates that even though participants are misestimating the number of cases, their responses are still rational to an extent.

Ten days later, on April $7^{\text {th }}$, we administered the online survey again to 787 people in the U.S. (398 new, 389 returning from the first study). This survey was identical to the first study except the presented data were updated to reflect the cumulative growth of COVID-19 cases in the U.S. from March $11^{\text {th }}$ to April $7^{\text {th }}$ (Fig. 1B). Note that these new data reveal a much more linear growth trend at the later dates in contrast to the more exponential trend evident at the time of Study 1.

On average, participants underestimated the number of COVID-19 cases in the U.S. on April $10^{\text {th }}\left[\mu_{o b s} \approx 475 \mathrm{k}\right.$, Truth $\left.\approx 503 \mathrm{k}\right]$ but overestimated the number of cases on April $13^{\text {th }}$ $\left[\mu_{o b s} \approx 601 \mathrm{k}\right.$, Truth $\left.\approx 587 \mathrm{k}\right]$ and April $16^{\text {th }}\left[\mu_{o b s} \approx 757 \mathrm{k}\right.$, Truth $\left.\approx 678 \mathrm{k}\right]($ Fig. $1 \mathrm{~B})$. Here we found that the Graph group predicted more cases than the Table group [Fig. 1B; $\beta_{\mathrm{T}-\mathrm{G}}=$ $\left.-24.6 \mathrm{k}, \mathrm{HDI}_{89 \%}=[-34.3 \mathrm{k},-15.2 \mathrm{k}], p d=100 \%\right]$. Although tables produced the most accurate estimates, people shown graphs were once again more confident in their predictions than people shown tables [Fig. 2B; $\left.\mathrm{CI}_{95 \%}=[-8.43,-4.05], p<0.001\right]$. These findings suggest that by April $7^{\text {th }}$, Americans began to overestimate the growth trajectory of COVID-19, however, tables again led to more accurate forecasting than graphs.

Despite the prima facie inconsistency of our two studies (graphs yielding lower estimates 20 in Study 1 and higher estimates in Study 2) one critical pattern was resilient: tables facilitated more accurate forecasts than graphs. To ensure that these effects were driven by our manipulation and not extraneous variables, we replicated both studies with a new cohort of 803 people and presented the same numerical data but replaced all mentions of the U.S. with references to a "hypothetical country". In this control study, we observed the same key findings from our first two studies. In the replication of Study 1 the graph group again forecasted fewer 
future cases $\left[\beta_{\mathrm{T}-\mathrm{G}}=11.8 \mathrm{k}, \mathrm{HDI}_{89 \%}=[5.07 \mathrm{k}, 18.5 \mathrm{k}], p d=99.7 \%\right]$. In our replication of Study 2 , the graph group forecasted more future cases $\left[\beta_{\mathrm{T}-\mathrm{G}}=-15.0 \mathrm{k}, \mathrm{HDI}_{89 \%}=\right.$ $[-29.9 \mathrm{k},-52.0 \mathrm{k}], p d=95.1 \%]$. In both our replication studies, the people who viewed tables of COVID-19 data produced more accurate forecasts of future growth compared to people who viewed graphs of the same data.

Why were the day-nine forecasts predominantly underestimations in Study 1, but overestimations in Study 2? Note that in Study 1, participants were presented with data that followed an obviously non-linear trend (true at the time of the spread of the disease; see Figure 1A), whereas in Study 2 participants were presented with data that followed a more linear trend (again, true at the time; see Figure 1B). In light of our successful replication of these data (Study 3), we reason that the behaviors observed in Studies 1 and 2 depended crucially on the structure and form of the presented data. For example, prior work has shown that people tend to overestimate when extrapolating from functions that appear more linear ${ }^{3}$ and that people underestimate positively accelerated trends (or "trend-dampen"). This underestimation increases as the slope of the trend increases ${ }^{6}$. Consistent with this research, we found that people underestimated future COVID-19 cases in Study 1 (where the slope of the trend was high) but not in Study 2 (where the slope of the trend was lower, and the function became more linear) ${ }^{7}$. The underlying causes of the differential effects of tables and graphs on forecasting are less clear, and prior research has yielded mixed results about such effects. But consistent with our findings, prior work has shown that people tend to underestimate exponential trends, especially when the trends are presented as graphs rather than tables ${ }^{1}$. For linear trends the opposite has been shown, namely that people tend to overestimate future data points, especially when the trends are presented in graphs rather than tables ${ }^{5}$. 
To summarize our key findings: people produced more accurate forecasts when given data in table rather than graph form, they systematically underestimated future cases and deaths during a time of growth well approximated by an exponential trend, and they overestimated cases and deaths during a time of growth well approximated by a linear trend. But to what extent 5 do people's forecasts actually relate to their attitudes about social distancing? 
A

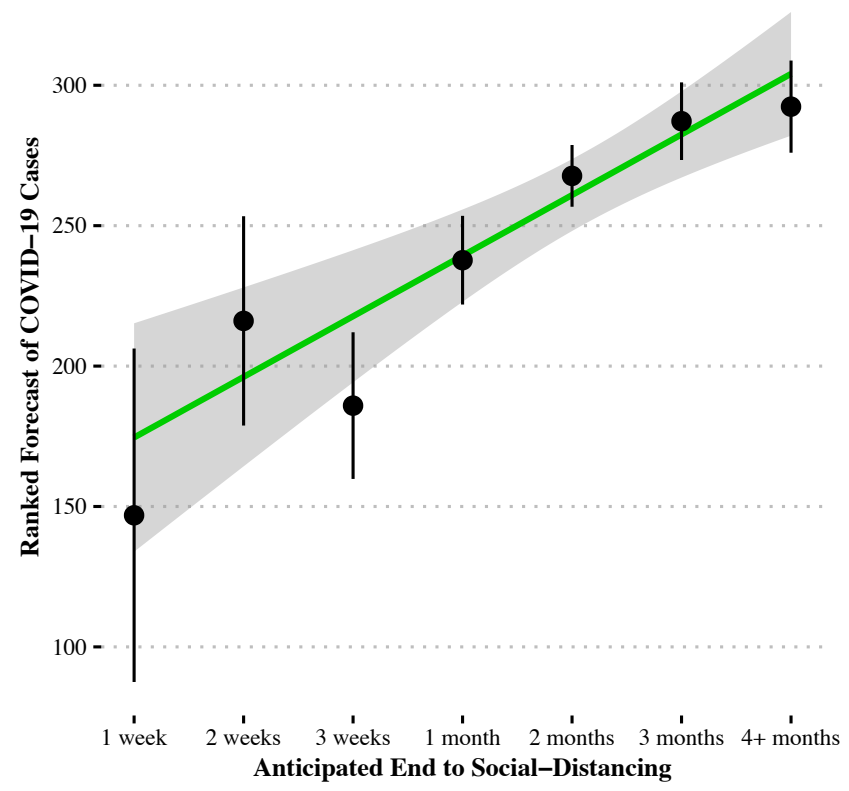

C

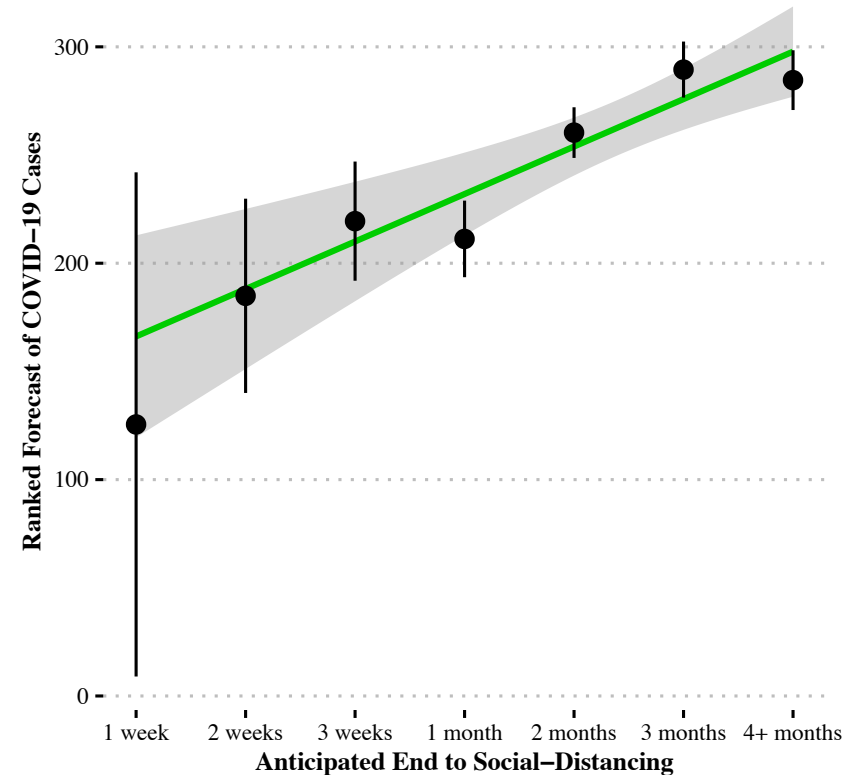

B

$400000-$

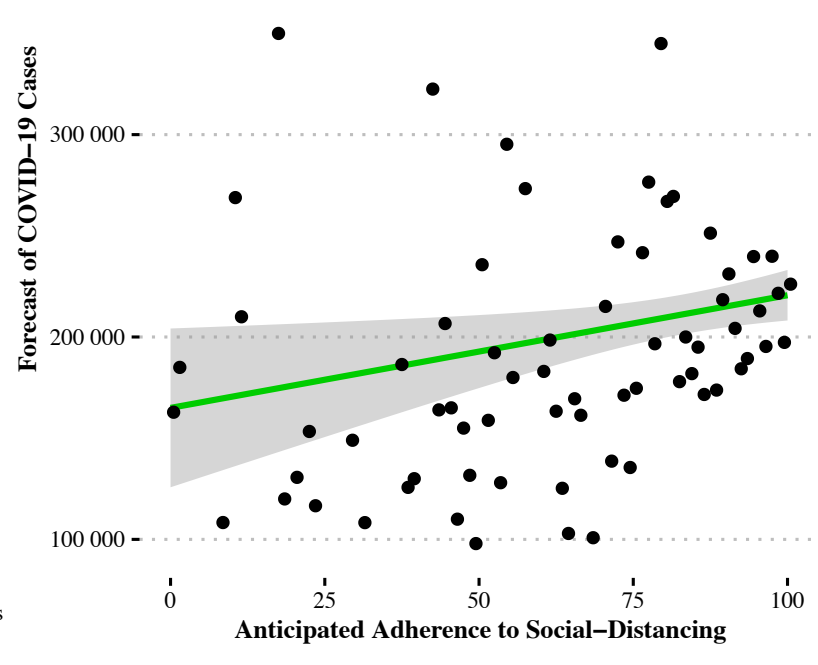

D

$1000000-$

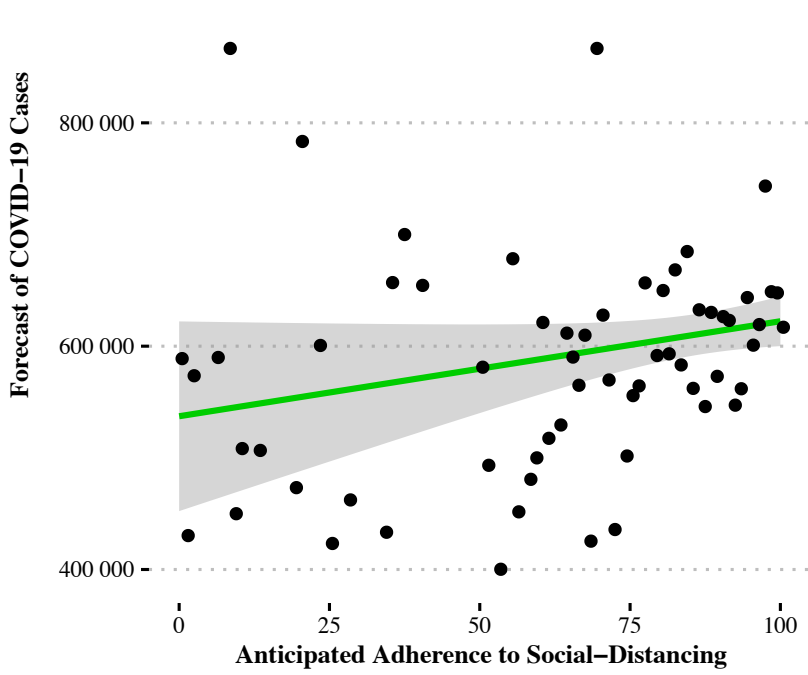

Figure 3. Relation between COVID-19 case forecasts and social-distancing forecasts. Panels A and B show data from Study 1 while Panels C and D show data from Study 2. In Panels $\mathbf{A}$ and $\mathbf{C}$, we plot means and standard errors of (ranked) COVID-19 case forecasts for each level of response to a question about how long social distancing will continue. Panels B and D show means of COVID-19 case forecasts for each level of response to a question about how well you will adhere to future social-distancing. Predictions from simple linear models are shown in green and included solely for illustrative purposes. For both Study 1 and Study 2, observed positive relationships between forecasted total cases of COVID-19 and both forecasted duration of social-distancing and anticipated adherence to social-distancing measures. 
To provide some insight into this question, we conducted a correlational analysis and observed a positive relationship between forecasted total cases of COVID-19 and forecasted time to desist from social-distancing both in Study 1 [Fig. 3A; $\tau=0.14, p=1.69 \times 10^{-5}$ ] and Study $2\left[\right.$ Fig. 3C; $\tau=0.13, p=5.54 \times 10^{-5}$ ]. In other words, the higher people's forecasts, the longer they expected social distancing orders to remain in place. In a second analysis, we observed a positive relationship between forecasted total cases of COVID-19 and anticipated adherence to future social-distancing measures both in Study 1 [Fig. 3B; $r=0.10, \mathrm{CI}_{95 \%}=[0.02,0.19], p=$ 0.02] and Study 2 [Fig. 3D; $\left.r=0.08, \mathrm{CI}_{95 \%}=[-0.01,0.16], p=0.08\right]$.

The implications of this work are straightforward. People grossly underestimate 10 positively accelerated growth functions in the case of disease incidence. So, merely presenting data to the public about a growth function of this sort does not guarantee that those data will be properly appreciated. But it is possible to increase this appreciation by considering the form in which the data are presented. We have explored just two cases here, tables versus static graphs, the most frequently used modalities in the popular media. And we found that even between these 15 two modalities, there are sizable differences in the levels of misestimation and self-reported confidence. This aligns with prior research showing that people are generally overconfident in their estimates ${ }^{4,7}$ but the finding that graphs lead to greater confidence provides a unique contribution to the forecasting literature. Finally, we found clear relationships between forecasts concerning cases and forecasts concerning social distancing, including personal adherence. So,

20 the form of the data themselves makes a difference in people's ability to forecast the future, and this ability to forecast may have important behavioral consequences through its link to attitudes toward social distancing. 
Our findings suggest that when choosing a mode of presentation for data, one must consider the shape of the data (type of trend) and the consequences of over and underestimating future data. For example, in the case of COVID-19, overestimation may lead to more caution, while underestimation may lead to less caution. In general, our findings present strong evidence 5 that basing estimates on data presented in tables leads to more accurate forecasting while basing estimates on static graphs leads to less accurate forecasting and false confidence in one's estimates. 


\section{References}

1. W. A. Wagenaar, S. D. Sagaria, Misperception of exponential growth. Percept Psychophys. 18, 416-422 (1975).

2. W. A. Wagenaar, H. Timmers, Extrapolation of exponential time series is not enhanced by having more data points. Percept Psychophys. 24, 182-184 (1978).

3. W. A. Wagenaar, H. Timmers, The pond-and-duckweed problem; Three experiments on the misperception of exponential growth. Acta Psychol. 43, 239-251 (1979).

4. M. R. Levy, J. Tasoff, Exponential-Growth Bias and Overconfidence. Ssrn Electron J (2015), doi:10.2139/ssrn.2574069.

5. S. Makridakis, R. J. Hyndman, F. Petropoulos, Forecasting in social settings: The state of the art. Int J Forecasting. 36, 15-28 (2020).

6. T. Wise, T. Zbozinek, G. Michelini, C. C. Hagan, D. Mobbs, Changes in risk perception and protective behavior during the first week of the COVID-19 pandemic in the United States. PsyArXiv (2020), doi:10.31234/osf.io/dz428.

15 7. N. Harvey, F. Bolger, Graphs versus tables: Effects of data presentation format on judgemental forecasting. Int J Forecasting. 12, 119-137 (1996).

8. N. Harvey, S. Reimers, Trend damping: Under-adjustment, experimental artifact, or adaptation to features of the natural environment? J Exp Psychology Learn Mem Cognition. 39, 589-607 (2012). 
9. R. M. Hogarth, S. Makridakis, Forecasting and Planning: An Evaluation. Manage Sci. 27, 115-138 (1981). 


\section{Methods}

\section{Study 1}

\section{Participants}

We recruited 817 participants from Amazon Mechanical Turk to participate in an online experiment ( $M$ Age: 37.8 , SD: 12.3; 56.2\% Male, 43.8\% Female). 525 subjects remained after applying our exclusion criteria (outlined below).

\section{Design}

Study 1 used a between-subjects design in which participants were randomly assigned to view news articles with COVID-19 data in either tabular or graphical from.

\section{Materials}

Two articles in the format of an online news article were created for the purpose of this experiment. Participants read a short vignette about the coronavirus in the United States. Participants viewed data on the total number of deaths and confirmed cases of COVID-19 in the U.S. in either a graphical format $(\mathrm{N}=242)$ or tabular format $(\mathrm{N}=283)$. The data shown to 15 participants were from the five weeks preceding the date of the study (March $27^{\text {th }}$ ). We limited the amount of data to five timepoints to strike balance between providing enough data to be useful for forecasting, but not too much data so as to overwhelm participants in the table groups.

Questionnaires. Participants were asked to estimate the number of confirmed cases, actual cases, and deaths, 3, 6, and 9 days after the article shown to them was written. They were 20 also asked to report their confidence in each of these nine estimates on a scale of 0-100.

After providing their estimates, demographic and individual difference data were collected on the following: age, gender, zip code, highest level of education, their mother's 
highest level of education, native language, risk aversion (9), conservatism (10), numeracy (11), and health status (12). Participants were also asked a number of COVID-19-related questions:

1. Estimate the probability that you will have contracted the illness within 9 days $(0-100 \%)$

2. Estimate the probability that you will have been hospitalized because of the illness within 9 days $(0-100 \%)$

3. Estimate the probability that you will have died from the illness within 9 days $(0-100 \%)$

4. How closely have you been following the news on the coronavirus? (Slider scale from 0 (Not at all) to 100 (Very closely))

5. How anxious are you about the current virus situation? (Slider scale from 0 (Not at all) to 100 (Extremely Anxious))

6. How successful have you been in engaging in social isolation? (Slider scale from 0 (Unsuccessful) to 100 (Very successful))

7. How successful will you be at engaging in social isolation in the next week? (Slider scale from 0 (Unsuccessful) to 100 (Very successful))

8. Estimate how much time will pass before we can stop social distancing. (1 week, 2 weeks, 3 weeks, 4 weeks, 2 months, 3 months, 4+ months)

9. Estimate the maximum number of new cases per day that will be reported in the US. (Free response)

All materials and questionnaires were administered using Qualtrics survey software.

Quality Assurance. To ensure data quality, participants were asked to verify that they were not a robot with a CAPTCHA at the beginning of the survey. We also included two attention check items: an embedded question in the risk aversion scale that asked participants to "please select 6" for the question and a free-response item that asked participants to report the 
name of the president of the U.S. In addition, we asked participants to self-report their perceived effort on the survey on a scale of 1-10. Participants were told that their rating would not affect their compensation for their participation.

\section{Procedure}

Participants located in the United States were invited to a survey via Amazon Mechanical Turk. They were told that they would read news articles and predict health-related data. After agreeing to participate, they were sent to a Qualtrics survey where they provided informed consent. They were next shown the news article with either the graphical or tabular display of data and were immediately asked to report their estimates and confidence for the number of confirmed cases and deaths 3, 6, and 9 days later. Participants were thanked and compensated $\$ 1$ after survey completion. All procedures were approved by the University of Michigan Institutional Review Board.

\section{Study 2}

\section{Participants}

We recruited 786 participants from Amazon Mechanical Turk to participate in an online experiment (M Age: 38.7, SD: 11.9; 53.6\% Male, 46.4\% Female). 524 subjects remained after applying our exclusion criteria (outlined below).

\section{Design}

Study 2 used a 2 (data visualization: graph, table) x 2 (prior participation: participated in

20 Study 1, new participant) factorial design, in which participants were again randomly assigned to data visualization conditions. Approximately half of participants participated in Study $1(\mathrm{~N}=$ $267)$ and half of participants $\operatorname{did} \operatorname{not}(\mathrm{N}=257)$.

\section{Materials}


The materials were the same as in Study 1, except the five data points presented to participants were for the five weeks preceding April $7^{\text {th }}$ instead of March $27^{\text {th }}$ (Study 1).

\section{Procedure}

All procedures were consistent with Study 1.

\section{Study 3}

\section{Participants}

We recruited 803 participants from Amazon Mechanical Turk to participate in an online experiment ( $M$ Age: 38.5, SD: 12.0; 57.1\% Male, 42.9\% Female). 429 subjects remained after applying our exclusion criteria (outlined below).

Study 3 used a 2 (timepoints: Study 1 data, Study 2 data) x 2 (data visualization: graph, table) factorial design. Participants were randomly assigned to view the data from Study $1(\mathrm{~N}=$ 207) or the data from Study $2(\mathrm{~N}=222)$ and were also randomly assigned to view those data in either graphical $(\mathrm{N}=89$, Study 1 materials; $\mathrm{N}=98$, Study 2 materials $)$ or tabular form $(\mathrm{N}=118$,

15 Study 1 materials; $\mathrm{N}=124$, Study 2 materials).

\section{Materials}

All materials and questionnaires were the same as those in Studies 1 and 2.

\section{Procedure}

All procedures were consistent with Studies 1 and 2 except participants were 20 compensated $\$ 0.75$ instead of $\$ 1$ after survey completion.

\section{Study 4}

\section{Participants}


We recruited 805 participants from Amazon Mechanical Turk to participate in an online experiment ( $M$ Age: 37.8, SD: 12.5; 50.7\% Male, 49.3\% Female). 467 subjects remained after applying our exclusion criteria (outlined below).

\section{Design}

Study 4 used a 2 (data visualization: graph, table) x 2 (data type: pure exponential, pure linear) factorial design. Participants were randomly assigned to make forecasts for exponential data $(\mathrm{N}=226)$ or linear data $(\mathrm{N}=241)$ and were randomly assigned to view those data in either graphical $(\mathrm{N}=97$, exponential; $\mathrm{N}=104$, linear $)$ or tabular form $(\mathrm{N}=129$, exponential; $\mathrm{N}=137$, linear). Materials

All materials and questionnaires were the same as those used in Studies 1 and 2 except that the presented data were five data points for either hypothetical exponential or hypothetical linear functions.

\section{Procedure}

All procedures were consistent with Study 3.

\section{Data Analysis Methods for Studies 1-4}

\section{Exclusion criteria}

We excluded participants who:

- failed the basic attention check ("Select 6")

- failed to identify the U.S. President as Donald Trump.

- were younger than 18 or older than 100 .

- estimated a decrease in total cases at any time point

- reported investing effort of less than 5 out of 10 
- took less than 30 seconds or more than 10 minutes to respond to the article stimuli

- did not provide a valid zip code

Upon initial viewing of the data, we realized that even after applying the above exclusion criteria, many of participants' forecasts of future cases were unrealistically high—so much that it was nearly impossible to visualize the response distributions due to the egregious positive skew. We therefore excluded any forecasts that exceeded 10 times the total number of cases on the date of the study. This corresponded to roughly one million cases in the nine days following Study 1 and roughly three million cases in nine days following Study 2.s

\section{Modeling}

We modeled participants' forecasts of future total COVID-19 cases using hierarchical linear models. The models included fixed effects of delay (within-subject; 3, 6, 9 days future), group (between-subject; table, graph), and their interaction. The models allowed intercepts to vary randomly by State.

We implemented the model using the R-package $\{$ brms $\}$, an open-source package for 15 Bayesian multilevel modeling $(1,2)$. This package translates input models into the probabilistic programming language stan, which supports approximate Bayesian inference over model parameters using Markov Chain Monte Carlo (MCMC) sampling with the No-U-Turns Sampler $(\mathrm{NUTS})^{3}$.

Due to the extreme positive skewness of the outcome variable, we used a Gamma distributional family for the model rather than the default Gaussian family. To facilitate specification of priors, we rescaled our outcome variable by dividing by the number of cases at the date of the study, which relocated our data to a range of approximately zero to ten, instead of 
the default zero to over one million (of course, after fitting, we put the parameters back to the original scale for easier interpretation). Our model was specified as follows:

$$
\begin{aligned}
& y_{i} \sim \operatorname{Gamma}\left(\mu_{i}, \alpha\right) \\
& \log \left(\mu_{i}\right)=\beta_{0}+\beta X+\beta_{0}^{\text {state }}
\end{aligned}
$$

$\beta \sim \operatorname{Normal}(\mathbf{0}, 1)$

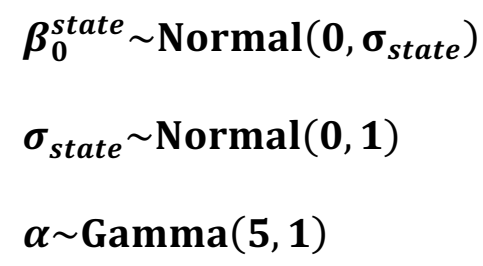

10 The first expression in the model specification above is the likelihood and the second expression is the regression formula with a log link function. In the regression formula, $\beta_{0}$ is the population intercept, $\beta_{0}^{\text {state }}$ is the state-wise 'random' intercept, $X$ denotes the predictors (delay, group, delay*group) and $\beta$ denotes the corresponding population-level regression coefficients. The auxiliary shape parameter of the gamma distribution is denoted by $\alpha$. The priors, indicated in 15 bold face, were intended to be weakly informative ${ }^{4}$.

All MCMC chains passed visual inspection, all $\hat{R}$ values were 1 , and all bulk and tail effective sample sizes (ESS) were large. After fitting the models, we performed graphical posterior predictive checks using the R packages $\{$ bayesplot $\}(5)$ and $\{100\}(6)$. To quantify uncertainty about the effects of interest, we computed $89 \%$ highest density intervals (HDI) as well as probabilities of direction $(p d)$. The HDI is defined such that, e.g., $89 \%$ of the distribution lies within the interval and every point inside the interval has higher credibility than every point outside the interval ${ }^{5}$. The $p d$ is defined as the probability that an effect goes in the direction indicated by the median estimate ${ }^{6}$. 


\section{References}

1. P.-C. Bürkner, brms : An R Package for Bayesian Multilevel Models Using Stan. Journal of Statistical Software. 80 (2017), doi:10.18637/jss.v080.i01.

2. P.-C. Bürkner, Advanced Bayesian Multilevel Modeling with the R Package brms. The R Journal. 10, 395 (2018).

3. B. Carpenter, A. Gelman, M. D. Hoffman, D. Lee, B. Goodrich, M. Betancourt, M. Brubaker, J. Guo, P. Li, A. Riddell, Stan : A Probabilistic Programming Language. Journal of Statistical Software. 76 (2017), doi:10.18637/jss.v076.i01.

4. A. Gelman, A. Jakulin, M. G. Pittau, Y.-S. Su, A weakly informative default prior distribution 10 for logistic and other regression models. Ann Appl Statistics. 2, 1360-1383 (2008).

5. J. Gabry, D. Simpson, A. Vehtari, M. Betancourt, A. Gelman, Visualization in Bayesian workflow. J Royal Statistical Soc Ser Statistics Soc. 182, 389-402 (2019).

6. A. Vehtari, A. Gelman, J. Gabry, Practical Bayesian model evaluation using leave-one-out cross-validation and WAIC. Stat Comput. 27, 1413-1432 (2017).

15 7. J. Kruschke, Doing Bayesian Data Analysis: A Tutorial with R, JAGS, and Stan. Academic press (2014).

8. D. Makowski, M. S. Ben-Shachar, S. H. A. H. Chen, D. Lüdecke, Indices of Effect Existence and Significance in the Bayesian Framework. Frontiers in psychology. 10, 2767 (2019). 


\section{Acknowledgements}

The authors thank the National Science foundation for funding this research (NSF \#2027822, awarded to the University of Michigan, J.J. as P.I.).

\section{Author Contributions}

M.F. and T.J.A. contributed to experimental design, data analysis, and manuscript preparation. J.J. and R.L. contributed to experimental design and manuscript preparation. A.B., M.Q., P.L., and P.S. contributed to experimental design and editing.

\section{Competing Interests Statement}

The authors declare no competing interests.

\section{Correspondence}

The authors to whom correspondence and material requests should be addressed are Madison

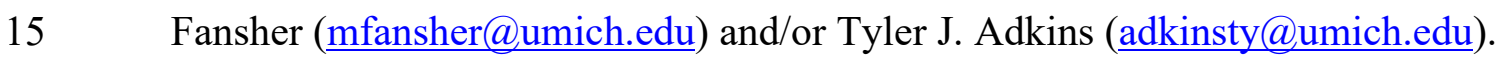

\section{Data Availability Statement}

All materials, data, and software will be made publicly available online prior to publication. 